\title{
Confiança, Valor Percebido e Lealdade em Trocas Relacionais de Serviço: Um Estudo com Usuários de Internet Banking no Brasil
}

\author{
Vinícius Andrade Brei \\ Carlos Alberto Vargas Rossi
}

\begin{abstract}
Resumo
O presente trabalho desenvolve e testa um modelo teórico baseado em hipóteses que analisam a relação entre a confiança, o valor percebido e a lealdade de usuários de Internet Banking em situações de trocas relacionais de serviço no Brasil. Uma pesquisa em todo o País com 611 usuários de Internet Banking de um grande banco de varejo e as análises através da técnica de Modelagem em Equações Estruturais validam, de forma geral, o modelo testado. As relações testadas no modelo levam à conclusão de que a confiança tem forte impacto no valor percebido pelos clientes que, por sua vez, também têm forte impacto na lealdade desses clientes à empresa. Valor foi identificado como uma variável mediadora parcial da relação entre confiança e lealdade. São apresentadas, ainda, conclusões acerca da atuação das empresas e sugestões para pesquisas futuras.
\end{abstract}

Palavras-chave: comportamento do consumidor; confiança; valor; lealdade; serviços.

\section{Abstract}

This paper is aimed on the development and test of a theoretical model based on hypotheses that analyze end-customers' trust, perceived value and loyalty on Internet Banking services relational exchanges in Brazil. Tested model is validated using structural equation modeling based on survey results with 611 Internet Banking end-customers of a major brazilian retail bank. Tested hypotheses show that trust has a strong impact on customers' perceived value which also has a strong impact on customers' loyalty. Perceived value was identified as a partial mediator variable on the trustloyalty relationship. Managerial and future research suggestions are also presented.

Key words: consumer behavior; trust; value; loyalty; services. 


\section{INTRODUÇÃO}

O objeto principal de estudo da ciência de marketing é conceituado como trocas (BAGOZZI, 1975); de acordo com essa visão, marketing é planejado e implementado para facilitar a troca de produtos por dinheiro (GRONRÖOS, 2000). Um caminho natural ao longo da análise das trocas é o estudo de como relações fortes e longos processos de trocas relacionais entre empresas e consumidores individuais são construídos e mantidos. O estudo destes processos é também chamado marketing de relacionamento.

Nas trocas relacionais com consumidores, quase sempre há uma assimetria presente, dado que a companhia tem poder econômico mais forte na relação. Embora inevitável, esta assimetria pode ser minorada pelas empresas; caso contrário, uma relação longa e duradoura dificilmente será alcançada. Um dos pontos-chave para minorar este efeito assimétrico em trocas relacionais de serviços é obter a confiança do consumidor (SIRDESHMUKH et al., 2002); outros autores também têm conceituado confiança como ponto-chave ou alicerce de trocas relacionais (DWYER; SCHURR; OH, 1987; GARBARINO; JOHNSON, 1999; MORGAN; HUNT, 1994).

O foco da maioria dos estudos sobre confiança tem sido em relacionamentos entre empresas, tendo sido pouco estudado o impacto desse comportamento nos relacionamentos entre empresas e clientes finais. Além disso, menos atenção ainda tem sido dada às conseqüências da confiança em relações de comércio eletrônico (SIRDESHMUKH et al., 2002), até mesmo porque a Internet é um canal de marketing relativamente novo para quase todas as empresas. Este trabalho busca suprir esta lacuna, desenvolvendo hipóteses que resultarão num modelo teórico voltado à explanação dos relacionamentos entre a confiança, o valor percebido e a lealdade dos clientes em situações de trocas relacionais com consumidores finais que utilizam o comércio eletrônico pela Internet para a compra de serviços.

Dessa maneira, em razão de 1) os clientes realizarem suas pesquisas on-line e decisões de compra com base na confiança (URBAN et al., 2000); 2) muitos problemas poderem acontecer em transações pela Internet; e 3) a confiança trazer benefícios para as companhias que nutrem relações duradouras, como lealdade e cooperação (GARBARINO; JOHNSON, 1999), a confiança tem lugar chave não só nas estratégias de trocas relacionais das empresas de serviço, mas em todas as companhias que usam a Internet como canal de marketing. 
Cabe, então, avaliar quais as conseqüências comportamentais da confiança nas empresas de serviço por parte dos clientes finais que usam o comércio eletrônico para aquisição desses serviços e suas conseqüências para a construção de relacionamento com essas empresas.

Entender as conseqüências da confiança por parte desses usuários de comércio eletrônico pode ainda ajudar as empresas a terem respostas para outras perguntas importantes relacionadas a estes investimentos, como: Os investimentos em Internet dão retorno?, Quais as conseqüências do uso da Internet para o relacionamento com o cliente?, O consumidor está mais satisfeito ou fiel às empresas em razão do uso da Internet?, Quais as diferenças em termos de confiança na empresa por parte de clientes com pouco e longo tempo de relacionamento?.

Embora já pesquisada em campos como sociologia, psicologia e comportamento organizacional e até mesmo em marketing de relacionamento, o estudo da confiança em relações de comércio eletrônico ainda não recebeu muita atenção, mesmo que alguns a considerem como: "a ferramenta de marketing de relacionamento mais poderosa disponível para uma empresa” (BERRY, 1996, p. 242) e "o marketing baseado na confiança como chave para o sucesso na Internet" (URBAN et al. 2000, p. 40).

\section{Confiança - Definição}

A literatura sobre confiança no ambiente de empresas e na relação destas com seus clientes tem por base inúmeros estudos provenientes de diversas áreas do conhecimento, o que gerou variadas definições sobre o que é o construto confiança (SINGH; SIRDESHMUKH, 2000). Por exemplo, enquanto os economistas tendem a ver a confiança como calculada ou institucional, os psicólogos normalmente dividem suas análises de confiança em termos de atributos das partes em uma relação, focando nas cognições que atributos pessoais geram. Uma das definições seminais do construto confiança é oriunda justamente da psicologia e foi elaborada por Rotter (1967) como sendo "a expectativa generalizada mantida por um indivíduo ou um grupo de que a palavra, promessa, verbal ou escrita, de outro indivíduo ou grupo pode ser confiada” (p. 651). Já os sociólogos vêem a confiança em propriedades socialmente inseridas nas relações entre as pessoas (ROUSSEAU et al., 1998).

Mesmo havendo divergências sobre a definição do construto confiança entre as diversas disciplinas, identifica-se pelo menos um ponto em comum a todas as 
áreas: as condições que devem existir para que a confiança seja desenvolvida. A primeira é risco, ou seja, a probabilidade de perda, quando interpretada por uma das partes decisoras; a segunda é interdependência, onde os interesses de uma parte não podem ser atingidos sem a colaboração de outra (ROUSSEAU et al., 1998).

Conceitos utilizados na área de marketing procuram englobar as duas condições acima citadas, onde se verifica que a confiança pode ser descrita como "a expectativa de uma parte de que a outra parte se comportará de uma maneira previsível em uma dada situação” (GRONRÖOS, 2000, p. 37). Outra definição usualmente citada é "tendência a acreditar num parceiro de negócios no qual se tem crédito” (MOORMAN et al., 1993, p. 82).

A dificuldade em não se ter uma única definição para o construto entre as diversas disciplinas motivou Rousseau et al. (1998) a proporem uma definição consensual. Para eles, “confiança é um estado psicológico que compreende a intenção de aceitar uma vulnerabilidade baseada em expectativas positivas das intenções ou comportamentos de outro" (p. 395).

Essa definição apresenta duas partes distintas. Na primeira, confiança é relacionada com expectativas (positivas) sobre as intenções e/ou comportamentos do parceiro de trocas. Essa conceituação é focada na crença de uma das partes de que a outra agirá de maneira responsável, evidenciando integridade e que não seja prejudicial à outra parte; esta é a chamada conceituação de confiança baseada na expectativa (SINGH; SIRDESHMUKH, 2000). Na segunda parte, a confiança é relacionada ao parceiro de trocas, aceitando-se a vulnerabilidade contextual; essa definição é a chamada confiança comportamental e referese à tendência de ação de uma parte em relação à outra.

Essa separação conceitual é também corroborada por Kramer (1999), através da distinção do construto em duas vertentes: confiança como um estado psicológico e como uma escolha de comportamento, congruente, portanto, com a bipartição acima citada. Esta última vertente é a base para a análise do presente estudo.

\section{A Confiança em Trocas Relacionais com Consumidores Finais}

Buscando a definição do que seria central para o conceito de marketing de relacionamento, Morgan e Hunt (1994) concluíram que deve ser algo que distingue trocas relacionais produtivas e efetivas das improdutivas e não efetivas. Assim, 
mesmo sabendo-se que inúmeros fatores contextuais podem contribuir para o sucesso ou fracasso de um relacionamento específico, os autores concluíram que confiança e comprometimento são conceitos centrais e essenciais para o sucesso de um marketing de relacionamento.

Em relações de trocas com consumidores finais, três fatores afetam o conceito de confiança. Em primeiro lugar, fatores situacionais e contextuais tendem a determinar a relevância da confiança nas trocas relacionais; em outras palavras, confiança não é um ingrediente necessário para a consumação de trocas entre clientes e empresas. Por outro lado, a falta de confiança também não evita que ocorra uma troca (SINGH; SIRDESHMUKH, 2000); ou seja, a importância da confiança (grau mínimo necessário) varia de acordo com a situação.

Mais especificamente, trocas onde a confiança é relevante são caracterizadas por: a) alto nível de ambigüidade de performance do provedor do serviço (ou seja, as avaliações do consumidor sobre o desempenho do prestador do serviço variam muito conforme a situação); b) as conseqüências são importantes (isto é, a performance do serviço traz resultados significativos para o valor entregue ao cliente - o conceito valor será mais bem explorado posteriormente); e c) maior interdependência (quando o consumidor participa do processo de troca) (SINGH; SIRDESHMUKH, 2000).

Em segundo lugar, uma especificação conotativa tende a influenciar a conceituação da confiança, ou seja, a especificação dos atributos com nível apropriado de precisão para que o construto tenha significado em múltiplos contextos. Em outras palavras, se um consumidor afirma 'eu confio no meu provedor de serviços', faltam atributos para avaliar não só qual é o grau de confiança desse consumidor, mas também diferenciar esse grau em relação ao de outros consumidores. A maneira encontrada para tratar esse problema foi o estabelecimento de atributos (também denominados antecedentes ou dimensões) que expliquem o significado do construto confiança.

Por último, a confiança é um continuum linear que tem num extremo a confiança e no outro a desconfiança, ambos qualitativamente diferentes e mantidos por mecanismos também distintos. Por exemplo, Sitkin e Roth (apud SINGH; SIRDESHMUKH, 2000) demonstraram empiricamente que numa relação entre empresa-empregado, expectativas não cumpridas em confiabilidade de tarefas geram violações de confiança, ao passo que incongruência de valores gera desconfiança. Tendo sido analisadas as principais definições de confiança e como esta influencia as trocas relacionais com consumidores, cabe então avaliar quais são as conseqüências mais importantes da confiança segundo a literatura. 


\section{Conseqüências da Confiança - Lealdade}

O estudo da perspectiva comportamental referida como lealdade surgiu nos anos 70 após um período em que era medida apenas como um padrão de compras repetidas. Em função de pesquisas dessa época terem apontado que propriedades estatísticas dos elementos determinantes de ciclos de compras repetidas eram estocásticas, ou seja, continham um componente aleatório não-analisável, os pesquisadores passaram a descrever a lealdade em função de explicações comportamentais (OLIVER, 1997).

Buscando reunir conceitos existentes na literatura e explorar aspectos cognitivos, afetivos e comportamentais, Oliver (1999) propôs a seguinte definição para lealdade: "um profundo compromisso mantido de recomprar... um produto/serviço preferido consistentemente no futuro, causando, portanto, compras repetidas da mesma marca ou mesmo conjunto de marcas, sem a preocupação com influências situacionais e esforços de marketing com potencial para causar mudanças de comportamento" (p. 34).

A conceituação da lealdade com base nos aspectos acima citados (compromisso de recomprar, de maneira consistente e sem a preocupação com fatores situacionais) pode ser relacionada com a idéia de que a atitude dos consumidores de tornarem-se leais passa por diversas fases: iniciando com a lealdade cognitiva, seguida pela afetiva e a conativa, terminando com a mais forte de todas, a chamada inércia de ação (OLIVER, 1999). Em função das vantagens de se ter a lealdade do consumidor, uma preocupação gerencial e acadêmica recente vem sendo a identificação daquilo que geraria a lealdade.

\section{O que Gera Lealdade: Satisfação, Qualidade do Serviço ou Confiança?}

O desenvolvimento e solidificação do conceito do marketing de relacionamento e várias das idéias a ele relacionadas, como a existência de um continuum de relacionamento entre a empresa e seus clientes (indo de orientações transacionais até relacionais) trouxe ainda mais força à identificação e busca de quais fatores explicariam determinados comportamentos dos clientes.

Acredita-se que o processo de decisão do consumidor em relação às empresas seja guiado por boa parte dessas variáveis latentes, denominadas de construtos 
mentais de ordem superior, como satisfação do consumidor, qualidade percebida de serviço, valor percebido, confiança e comprometimento. Considera-se que essas avaliações globais sumariam o conhecimento e experiência dos consumidores e guiam as ações subseqüentes deles em relação a uma empresa. Por muito tempo, considerou-se que o construto-chave para a predição de comportamentos leais dos consumidores fosse a satisfação (GARBARINO; JOHNSON, 1999; SZYMANSKI; HENARD, 2001); até que estudos sobre serviços incluíram a qualidade (PARASURAMAN; GREWAL, 2000) e trabalhos sobre marketing de relacionamento incluíram a confiança e compromisso como fatores-chave para a predição desses comportamentos (MORGAN; HUNT, 1994; MOORMAN et al., 1993). Essa diversidade estimulou alguns autores a tentarem identificar qual construto seria o maior responsável pela lealdade.

Buscando analisar relações entre satisfação e lealdade, Oliver (1999) verificou que a satisfação é um estado temporal pós-uso decorrente de uma situação de consumo única ou de experiências repetidas que refletem como um produto ou serviço supriu seu propósito. A partir da perspectiva da empresa, a satisfação é entregue ao consumidor. Lealdade, ao contrário, é um estado de preferência duradouro obtido a partir de uma postura determinada do cliente para com a empresa. Oliver conclui afirmando que a satisfação não se transforma em lealdade sem a existência de outros fatores como determinação pessoal e suporte social; assim, sem a existência desses fatores, um consumidor pode continuar satisfeito, mas não ir além desse estado (p. 42).

Ao tentar identificar os papéis de satisfação, lealdade e confiança e suas conseqüências em trocas relacionais, Garbarino e Johnson (1999) concluíram que diferentes fatores mediam as intenções futuras de clientes com fracos e fortes relacionamentos com as empresas (também denominados clientes transacionais e relacionais). A primeira importante conclusão é de que a satisfação geral determina as intenções futuras de clientes com pouco relacionamento com a empresa. Já para os clientes relacionais, os grandes responsáveis pelas intenções futuras são a confiança e comprometimento. Assim, programas de marketing transacionais baseados na gestão da satisfação seriam mais efetivos para clientes com pouco relacionamento, ao passo que programas de marketing de relacionamento voltados para clientes relacionais deveriam ter como foco a construção e manutenção da confiança e do comprometimento, e não da satisfação.

Sirdeshmukh et al. (2002) ratificaram o trabalho de Garbarino e Johnson (1999), testando a relação entre confiança e lealdade em trocas relacionais. Aqueles autores verificaram que a relação entre confiança e lealdade é baseada em argumentos de reciprocidade. Quando um provedor de serviço age de maneira a 
construir a confiança do consumidor, o risco percebido tende a diminuir e permite que o consumidor faça predições mais confiantes sobre os comportamentos futuros dessa empresa. Para manter essa confiança, estudos sobre reciprocidade sugerem que os consumidores tendem a agir de maneira cooperativa diante do provedor de serviço, oferecendo evidências comportamentais de lealdade (GASSNHIEMER et al., 1998).

Sirdeshmukh et al. (2002) verificaram, ainda, que a confiança impacta a lealdade através da mudança de percepção dos consumidores sobre a congruência de valores com o provedor do serviço. O aumento da confiança impacta a similaridade de valores entre o cliente e a empresa e, conseqüentemente, aumenta o envolvimento daqueles clientes no relacionamento; assim, o crescimento do envolvimento entre as partes promove a reciprocidade e contribui para o compromisso relacional.

Algumas avaliações globais de clientes, como satisfação geral, qualidade percebida de serviço e valor percebido, ocasionalmente apresentam forte relação estatística, descrita como efeito halo ou multicolinearidade (GARBARINO; JOHNSON, 1999). Compromisso e lealdade, da mesma forma que satisfação geral, são avaliações globais e, portanto, também suscetíveis ao efeito halo. Morgan e Hunt (1994), num dos principais trabalhos sobre variáveis-chave no marketing de relacionamento, referem-se a esses dois construtos de maneira intercambiada, citando que “...eles também identificaram que confiança leva a níveis mais elevados de lealdade (isto é, comprometimento)...” (p. 24).

De maneira diferente, a confiança tem sido analisada majoritariamente como um antecedente e não um sinônimo de lealdade (DONEY; CANNON, 1997; GEINSKENS et al., 1998; SINGH; SIRDESHMUKH, 2000, SIRDESHMUKH et al., 2002). Ganesan e Hess (1997, p. 440) defendem que "a conseqüência mais freqüentemente examinada da confiança é o comprometimento com um relacionamento”. Dick e Basu (1994, p.108) ratificam essa idéia afirmando que "confiança no provedor de serviço é freqüentemente a chave para lealdade".

Da mesma forma que Sirdeshmukh et al. (2002), este trabalho relaciona apenas a confiança e não satisfação, qualidade do serviço e comprometimento como antecedentes da lealdade em situações de trocas relacionais, uma vez que a revisão teórica realizada identificou que há mais suporte na literatura para os antecedentes de lealdade aqui identificados. Dessa forma, propõe-se que:

\section{$\mathrm{H}_{1}$ : A lealdade do cliente é positivamente influenciada pela confiança.}


Estudos mais recentes, entretanto, têm indicado a existência de uma variável mediadora na relação entre confiança e lealdade: o valor percebido pelo cliente na relação de troca (SINGH; SIRDESHMUKH, 2000; SIRDESHMUKH et al., 2002).

\section{Conseqüências da Confiança - Valor}

O valor que um cliente enxerga numa troca atua como um mecanismo mediador do efeito da confiança na lealdade (SIRDESHMUKH et al., 2002). O conceito mais utilizado de valor percebido dentro da literatura de marketing é o de Zeithaml (1988): “valor percebido é a avaliação geral pelo consumidor da utilidade de um produto baseado em percepções do que é recebido e do que é dado" (p. 14). Aplicando esse conceito para situações de prestação de serviços relacionais, Sirdeshmukh et al. (2002) afirmam que valor é definido como a percepção do consumidor dos benefícios menos os custos de manutenção de um relacionamento contínuo com um provedor de serviço (p.21).

Sirdeshmukh et al. (2002) verificaram ainda que o papel mediador de valor nas trocas relacionais é embasado nas teorias de identificação de metas e ações que, juntas, propõem que: a) as ações do consumidor são guiadas ou (identificadas) pelas metas que elas buscam atingir; b) metas múltiplas e às vezes conflitantes podem ocorrer; c) as metas são organizadas hierarquicamente como metas superiores e inferiores; e d) os consumidores regulam suas ações para atingir metas superiores. Esses autores afirmam que metas superiores são estados finais desejados, enquanto metas inferiores desempenham papéis instrumentais (p. 21).

A existência de confiança num parceiro de negócios e o compromisso com aquele parceiro, portanto, é mais importante para os clientes que vêem mais valor no próprio relacionamento. Estes clientes mais voltados para o relacionamento apreciam mais a existência de confiança e compromisso do que estarem satisfeitos com cada uma das trocas de um relacionamento contínuo; já os clientes mais transacionais demandam que toda transação deva ser satisfatória (GRONRÖOS, 2000, p. 39).

Outras relações de lealdade com seus antecedentes já foram analisadas teórica (NEAL, 1999; VANDERMERWE, 2000) e empiricamente (CHANG; WILDT, 1994; GRISAFFE; KUMAR 1998; SIRDESHMUKH et al., 2002), identificando o papel mediador do valor na literatura, mais especificamente na relação entre 
qualidade de serviço-lealdade. Parasuraman e Grewal (2000) propuseram um modelo teórico não testado empiricamente; já Chang e Wildt (1994) identificaram esse papel mediador em duas indústrias diferentes. Grisaffe e Kumar (1998) concluíram que, apesar de estatisticamente significante, valor é um mediador parcial, e não total, da relação entre qualidade de serviço-lealdade.

De maneira semelhante a Grisaffe e Kumar (1998) no estudo da relação entre qualidade de serviço-lealdade, Sirdeshmukh et al. (2002) identificaram que existe a mediação de valor na relação confiança-lealdade, porém também verificaram resultados estatisticamente significantes na relação direta em uma das duas indústrias analisadas (sem a mediação de valor). Assim, verifica-se ser mais prudente incluir no modelo proposto neste trabalho tanto a relação mediadora de valor entre confiança-lealdade (hipótese mais consistente na literatura), como a relação direta entre confiança-lealdade (esta última já explicitada na Hipótese $\left.\mathrm{H}_{1}\right)$. Dessa forma, propõe-se que:

\section{H2: O valor percebido pelo cliente é positivamente influenciado pela confiança. \\ H3: Valor percebido é uma variável mediadora da relação confiança- lealdade}

Finalmente, espera-se que um cliente apresente intenções comportamentais de lealdade em relação a um provedor de serviço, na medida em que as trocas relacionais com a empresa provejam mais valor a ele. Levando-se em consideração que valor é uma meta superior e lealdade um comportamento eventual, que depende de algumas condições prévias para que se manifeste (supõe-se que ninguém será fiel a uma empresa apenas pelo ato em si, mas pela busca de alguma vantagem oriunda desse comportamento), verifica-se que o valor regula as ações do consumidor em relação à empresa. No caso do presente trabalho, a relação testada é entre confiança e lealdade (SIRDESHMUKH et al. 2002). Assim, elabora-se a última hipótese deste trabalho:

H4: A lealdade do consumidor em relação ao provedor de serviços é positivamente influenciada pelo valor percebido.

Após a construção de todas as hipóteses, cabe, então, apresentar o modelo que resume essas proposições: 
Figura 1: Modelo Teórico da Relação entre Confiança, Valor e Lealdade

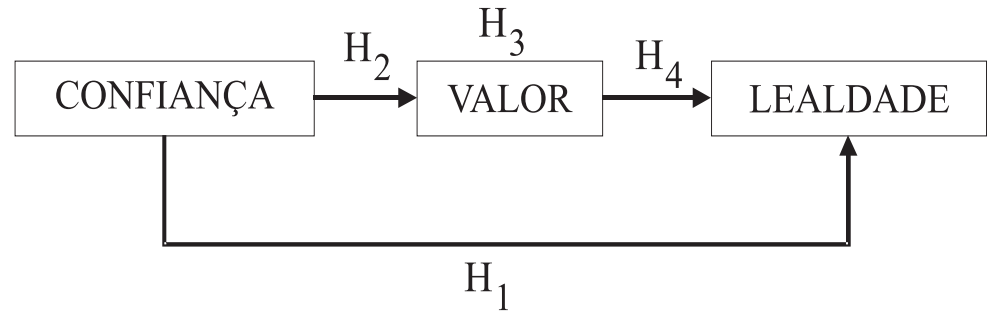

\section{Método}

Para realizar o teste do modelo proposto foi realizada uma pesquisa de campo em todo o Brasil com clientes de um grande banco de varejo brasileiro; o trabalho foi desenvolvido em duas fases distintas: uma qualitativa e outra quantitativa. A fase qualitativa teve por objetivos a elaboração e pré-teste do questionário utilizado na fase quantitativa, utilizado para se testar as hipóteses anteriormente apresentadas.

Dois métodos foram utilizados durante a fase qualitativa da pesquisa para viabilizar a elaboração do questionário: a revisão da literatura e pesquisa com informantes-chave. A revisão de literatura serviu para a identificação de itens e escalas a serem utilizadas para medição e validação dos construtos confiança, valor, lealdade e satisfação transacional.

A avaliação multifacetada de serviços tem diversos precedentes na literatura (CROSBY; STEPHENS, 1987; DONEY; CANNON, 1997; SINGH, 1991; SIRDESHMUKH et al. 2002). Por isso a escala de medição da confiança adaptada do trabalho de Sirdeshmukh et al. (2002) ${ }^{(1)}$ foi aplicada na avaliação da confiança em diversas facetas de contato do cliente com a empresa - empregados de fronteira (EF), políticas e práticas gerenciais (PPG) e, como este trabalho avalia trocas realizadas por usuários de Internet Banking, também a confiança na faceta web-site (WS).

A escala de valor foi adaptada de trabalhos anteriores sobre o assunto (GRISAFFE; KUMAR, 1998; DODDS et al., 1991); já a de lealdade foi adaptada dos trabalhos de Murray (1991), Sirdeshmukh et al. (2002) e Zeithaml et al. (1996). A inclusão da escala utilizada para medir satisfação transacional (a ser explicada posteriormente) foi adaptada do trabalho de Sirdeshmukh et al. (2002).

O pré-teste do instrumento utilizado no presente trabalho foi realizado durante os meses de janeiro a março de 2001, primeiramente através de entrevistas 
pessoais e, posteriormente, por telefone. Foram realizadas 21 entrevistas com pessoas com as mesmas características da amostra desejada.

A lista de clientes entrevistados na fase quantitativa foi sorteada pelo próprio sistema administrador da base de dados da empresa objeto da pesquisa, dentre todos os clientes usuários de Internet Banking, usando-se o critério probabilístico.

A técnica de Modelagem em Equações Estruturais - MEE foi utilizada para análise dos dados e estimação do modelo proposto. Seguindo orientação de Hair et al. (1998), dois passos distintos da técnica MEE foram realizados para se obter uma representação acurada da confiabilidade dos indicadores utilizados; assim, primeiramente realizou-se uma análise fatorial confirmatória no modelo de mensuração para realização dos testes de validação fatorial dos construtos teóricos do modelo proposto. Ou seja, foi verificado se os itens operacionais utilizados para medir os construtos são significativos (através de suas cargas fatoriais) e se realmente medem aquilo que se esperava que medissem (isto é, se foi observada a validade de construto); e, ainda, se cada construto tem confiabilidade satisfatória, calculada através do alfa de Cronbach, para se estimarem as relações causais em etapas posteriores do teste do modelo.

A estimação dos modelos de mensuração e estrutural foi realizada com o software EQS, utilizando-se a técnica de estimação ERLS (Elliptical Reweighted Least Squares). Foram usadas as seguintes medidas de adequação para análise do modelo: qui-quadrado $\left(\chi^{2}\right)$, Standardized Root Mean Squared Residual (std. RMR), Root Mean Square Error of Approximation (RMSEA), Normed Fit Index (NFI) e Nonnormed Fit Index (NNFI) e Comparative Fit Index (CFI). Para avaliação da significância dos coeficientes estimados foram realizados testes- $t$ dos valores obtidos.

\section{Caracterização da Amostra}

Foram entrevistados por telefone 611 clientes usuários do Internet Banking em todo o Brasil durante o mês de abril de 2001, resultando numa taxa de resposta de pouco menos de $10 \%$. Para garantir que todos os respondentes usavam efetivamente a Internet para realização de suas operações bancárias, inseriu-se uma pergunta-filtro no início do questionário: “Em média, quantas vezes POR MÊS o(a) Sr.(a) realiza operações bancárias, usando a Internet no Banco X (qualquer tipo de operação: saldo, extrato, investimento, resgate, transferência, empréstimos, etc.)?” Se a resposta era “zero” ou nenhuma, o respondente não era entrevistado. 
Em termos geográficos, a subdivisão da amostra por região do Brasil em termos percentuais foi: 41\% Sudeste, 28\% Sul, 15\% Nordeste, 13\% Centro-Oeste e 3\% Norte, tendo o Estado de São Paulo apresentado o maior número de entrevistados (116 pessoas). A amostra apresentou-se predominantemente masculina (64\% de homens e $36 \%$ de mulheres). Cerca de 50\% da amostra era composta por pessoas casadas e $41 \%$ dos entrevistados eram solteiros. Cinco faixas etárias foram adotadas nesta pesquisa: até 20 anos (7,1\% dos entrevistados); de 21 a 30 anos (35,6\%); de 31 a 40 anos (25,2\%); de 41 a 55 anos (25,5\%); e mais de 55 anos (6,6\%). Houve um predomínio de pessoas com grau de instrução universitário completo e pós-graduação (55,3\%) e faixa salarial acima de 10 salários mínimos (55\%).

Todos os 611 clientes entrevistados tinham, pelo menos, três meses de relacionamento com a Empresa. A média, porém, foi bem superior a este período - mais de 57\% dos clientes tinham mais de 3 anos de relacionamento com a empresa, sendo a amostra bastante adequada para a avaliação das hipóteses acerca das relações entre confiança, valor e lealdade em situações de trocas relacionais de serviço.

Por fim, a avaliação da utilização do canal Internet expressa pelos próprios clientes indicou que $62,3 \%$ dos clientes utilizam o canal mais que cinco vezes por mês para realização de transações bancárias, sendo que 32,3\% utilizam 13 vezes ou mais por mês (ou mais do que três vezes por semana). Tendo sido caracterizada a amostra, cabe então a apresentação dos resultados do trabalho.

\section{Resultados}

O teste do modelo proposto foi feito em duas etapas, conforme sugerido por Hair et al. (1998): primeiramente a estimação do modelo de mensuração e, posteriormente, do modelo estrutural.

\section{Estimação do Modelo de Mensuração}

A validação dos contructos em análise através do modelo de mensuração seguiu alguns critérios sugeridos por Sirdeshmukh et al. (2002): a) o quanto o modelo teórico se ajustou aos dados coletados; b) a magnitude e significância estatística das cargas fatoriais; c) as confiabilidades e variâncias extraídas de cada fator; e d) as intercorrelações dos fatores produzirem evidência de validade discriminante. 
Para facilitar a estimação do modelo de mensuração, ele foi subdividido em dois sub-modelos: um que mediu a confiança (em cada uma das três facetas anteriormente citadas - cada uma medida por três itens); e outro que mediu valor, lealdade e satisfação. O primeiro submodelo apresentou os seguintes resultados:

\section{Tabela 1: Índices de Ajuste do Modelo S1}

\begin{tabular}{c|c|c|c|c|c|c|c}
\hline Modelo & $\chi^{\mathbf{2}}$ & g.l. & CFI & NFI & NNFI & std. RMR & RMSEA \\
\hline S1 & 108,359 & 24 &, 966 &, 957 &, 949 &, 055 &, 077 \\
\hline
\end{tabular}

Nota: segundo Hair et al. (1998), valores $\alpha$ acima de 0,70, acima de 0,90 para o CFI; 0,90 para o NFI; 0,90 para o NNFI; e valores abaixo de 0,05 para o Std. RMR e entre 0,05 e 0,08 para o RMSEA são considerados satisfatórios.

Tabela 2: Estatísticas dos Construtos Avaliados do Modelo S1

\begin{tabular}{|c|c|c|c|c|c|c|}
\hline \multirow{2}{*}{$\begin{array}{c}\text { Fatores } \\
\text { (confiabilidade }^{1} \text { ) } \\
\left.\text { [variância } \text { extraída }^{2}\right] \\
\end{array}$} & \multirow[b]{2}{*}{ Variáveis } & \multicolumn{2}{|c|}{$\begin{array}{l}\text { Coeficientes não- } \\
\text { padronizados }\end{array}$} & \multirow{2}{*}{$\begin{array}{c}\begin{array}{c}\text { Coeficientes } \\
\text { padronizados }\end{array} \\
\text { Cargas } \\
\text { Fatoriais } \\
\end{array}$} & \multirow[t]{2}{*}{ Valores $t^{3}$} & \multirow{2}{*}{$\begin{array}{l}\text { Correlações } \\
\text { entre os } \\
\text { construtos }\end{array}$} \\
\hline & & $\begin{array}{c}\text { Cargas } \\
\text { Fatoriais }\end{array}$ & $\begin{array}{l}\text { Erros- } \\
\text { padrão }\end{array}$ & & & \\
\hline \multirow{3}{*}{$\begin{array}{c}\text { Confiança no } \\
\text { WS (F1) } \\
(\alpha=, 79) \\
{[0,56]}\end{array}$} & V33 &, 424 &, 035 &, 712 & $12,276 * *$ & \multirow{9}{*}{$\begin{array}{l}F 1-F 2=, 45 \\
F 2-F 3=, 56 \\
F 2-F 3=, 69\end{array}$} \\
\hline & V34 & ,509 &, 043 & ,689 & $11,811^{* *}$ & \\
\hline & V5 &, 595 & 041 &, 826 & $14,495 * *$ & \\
\hline \multirow{3}{*}{$\begin{array}{c}\text { Confiança nos } \\
\text { EF (F2) } \\
(\alpha=, 83) \\
{[0,66]}\end{array}$} & V36 & ,564 & ,038 & ,790 & $14,672 * *$ & \\
\hline & V37 & ,706 & 041 & ,895 & $17,188 * *$ & \\
\hline & V38 & ,724 & ,054 & ,739 & $13,485^{* *}$ & \\
\hline \multirow{3}{*}{$\begin{array}{c}\text { Confiança nas } \\
\text { PPG (F3) } \\
(\alpha=, 87) \\
{[0,71]}\end{array}$} & V39 &, 672 &, 042 &, 840 & $16,137 * *$ & \\
\hline & V40 & ,729 &, 041 & ,901 & $17,755^{* * *}$ & \\
\hline & V41 &, 777 &, 053 & ,783 & $14,719^{* * *}$ & \\
\hline
\end{tabular}

Notas: (1) confiabilidade calculada pelo Alfa de Cronbach. Valores acima de 0,70 são satisfatórios.

(2) Variâncias extraídas acima de 0,50 indicam valores satisfatórios.

(3) Baseado em testes-t uni-caudais: *valores $\mathrm{t}>1,65 \Rightarrow \mathrm{p}<0,05$;

$* *$ valores $\mathrm{t}>2,33 \Rightarrow \mathrm{p}<0,01$.

Coeficientes significantes estão negritados.

Fonte das tabelas: análise de dados.

Como pode ser verificado nas tabelas acima, todos os índices de adequação do modelo S1 foram satisfatórios (com exceção do std. RMR, que ficou ligeiramente acima do valor considerado adequado). Bons índices também foram encontrados nos indicadores que medem a confiabilidade das variáveis analisadas (todos os $\alpha$ ficaram acima de 0,79 e todas as cargas fatoriais acima de 0,69 , valores bem acima do patamar considerado satisfatório). Os resultados do segundo submodelo podem ser observados nas Tabelas 3 e 4 . 
Tabela 3: Índices de Ajuste do Modelo S2

\begin{tabular}{l|c|c|c|c|c|c|c}
\hline \multicolumn{1}{c|}{ Modelo } & $\chi^{\mathbf{2}}$ & g.l. & CFI & NFI & NNFI & std. RMR & RMSEA \\
\hline S2 & 100,301 & 24 &, 974 &, 966 &, 961 &, 046 &, 072 \\
\hline
\end{tabular}

Fonte: análise de dados.

Tabela 4: Estatísticas dos Construtos Valor (F5), Lealdade (F6) e Satisfação (F7)

\begin{tabular}{|c|c|c|c|c|c|c|}
\hline \multirow{2}{*}{$\begin{array}{c}\text { Fatores } \\
\text { (confiabilidade) } \\
\text { [variância extraída] }\end{array}$} & \multirow[b]{2}{*}{ Variáveis } & \multicolumn{2}{|c|}{$\begin{array}{l}\text { Coeficientes não- } \\
\text { padronizados }\end{array}$} & \multirow{2}{*}{$\begin{array}{c}\begin{array}{c}\text { Coeficientes } \\
\text { padronizados }\end{array} \\
\text { Cargas } \\
\text { Fatoriais }\end{array}$} & \multirow[t]{2}{*}{ Valores $t$} & \multirow{2}{*}{$\begin{array}{c}\text { Correlações } \\
\text { entre os } \\
\text { construtos }\end{array}$} \\
\hline & & $\begin{array}{c}\text { Cargas } \\
\text { Fatoriais }\end{array}$ & $\begin{array}{l}\text { Erros- } \\
\text { padrão }\end{array}$ & & & \\
\hline \multirow{3}{*}{$\begin{array}{c}\text { Valor }(\mathrm{F} 5) \\
(\alpha=, 76) \\
{[0,51]}\end{array}$} & V42 &, 730 & ,064 &, 605 & $11,405 * *$ & \multirow{9}{*}{$\mathrm{F} 5-\mathrm{F} 6=, 81$} \\
\hline & V43 & ,643 & ,049 & ,686 & $13,234 * *$ & \\
\hline & V45 & ,729 & ,044 & ,828 & $16,501 * *$ & \\
\hline \multirow{3}{*}{$\begin{array}{l}\text { Lealdade (F6) } \\
\quad(\alpha=, 81) \\
{[0,60]}\end{array}$} & V46 & ,733 & 047 & ,775 & $15,704 * *$ & \\
\hline & V47 & 888 & 054 & 805 & $16,482 * *$ & \\
\hline & V49 & ,604 & ,040 & ,745 & $14,946 * *$ & \\
\hline \multirow{3}{*}{$\begin{array}{c}\text { Satisfação }(\mathrm{F} 7) \\
(\alpha=, 95) \\
{[0,87]}\end{array}$} & V50 & 1,178 & 055 & ,924 & $21,326 * *$ & \\
\hline & V51 & 1,282 & 059 & ,939 & $21,856 * *$ & \\
\hline & V52 & 1,130 & ,053 & ,929 & $21,503 * *$ & \\
\hline
\end{tabular}

Fonte: análise de dados.

Da mesma maneira que já ocorrera no modelo S1, os índices de adequação do modelo S2 também foram satisfatórios. Dessa maneira, tendo todas as análises apresentado resultados satisfatórios segundo os critérios estabelecidos, pode-se considerar os contrutos validados e adequados para a realização da etapa seguinte: estimação do modelo estrutural.

\section{Estimação do Modelo Estrutural}

A partir de sua validação, cada um dos construtos em análise foi transformado em uma variável observável, através da composição de itens. Por exemplo, o construto confiança passou a ser representado por apenas uma variável, composta pela média dos escores individuais de cada respondente de todas as variáveis utilizadas para medi-lo no modelo de mensuração.

A estimação do modelo estrutural também foi realizada com o uso da técnica MEE, com a ressalva de que, neste caso, foram realizadas Regressões Múltiplas, através do Diagrama de Caminhos (Path Diagram), do seguinte sistema de equações (representando as relações citadas na Figura 1):

$$
\begin{aligned}
& \mathbf{Y}_{1}=\beta_{01}+\beta_{11} \mathbf{X}_{1}+\beta_{21} \mathbf{S}_{1}+\boldsymbol{\varepsilon}_{1} \\
& \mathbf{Y}_{\mathbf{2}}=\beta_{02}+\beta_{12} \mathbf{X}_{2}+\beta_{22} \mathbf{Y}_{1}+\beta_{32} \mathbf{S}_{\mathbf{2}}+\boldsymbol{\varepsilon}_{2} \\
& \mathbf{Y}_{3}=\beta_{03}+\beta_{13} \mathbf{X}_{3}+\beta_{23} \mathbf{S}_{3}+\boldsymbol{\varepsilon}_{\mathbf{3}}
\end{aligned}
$$


Onde: $\mathbf{Y}_{1}$ representa Valor, $\mathbf{Y}_{2}$ e $\mathbf{Y}_{3}$ Lealdade, $\mathbf{X}_{1}$ a $\mathbf{X}_{3}$ confiança e $\boldsymbol{\varepsilon}_{1}$ a $\boldsymbol{\varepsilon}_{3}$ representam a mensuração do erro, que é um somatório dos erros de especificação e erro de mensuração aleatório. A variável dependente Lealdade foi repetida $\left(\mathbf{Y}_{2}\right.$ e $\left.\mathbf{Y}_{3}\right)$, para que fosse testada com e sem o efeito direto da variável valor $\left(\mathbf{Y}_{1}\right)$, isto para testar se a mediação de valor é parcial ou total na relação entre confiança e lealdade.

Incluiu-se, ainda, as variáveis $\mathbf{S}_{1}$ a $\mathbf{S}_{3}$ representantes da satisfação com o último encontro com a Empresa como variáveis independentes em cada uma das equações desenvolvidas. Essa inclusão decorre da observação de Peterson e Wilson apud Sirdeshmukh et al. (2002), de que consumidores mais satisfeitos tendem a ser super-representados em surveys, tornando os efeitos halo dessa satisfação significantes. Os resultados da estimação do modelo estrutural através das equações de regressões foram os seguintes:

\section{Tabela 5: Resultados das Regressões Múltiplas para Estimação do Modelo Estrutural}

\begin{tabular}{|c|c|c|c|c|c|}
\hline \multirow[t]{2}{*}{$\begin{array}{c}\text { Variáveis } \\
\text { Dependentes }\left(\mathbf{R}^{2}\right)\end{array}$} & \multirow[t]{2}{*}{ Variáveis Independentes } & \multicolumn{2}{|c|}{$\begin{array}{l}\text { Coeficientes não- } \\
\text { padronizados }\end{array}$} & \multirow{2}{*}{$\begin{array}{c}\begin{array}{c}\text { Coeficientes } \\
\text { padronizados }\end{array} \\
\beta\end{array}$} & \multirow[t]{2}{*}{ Valores $t$} \\
\hline & & b & $\begin{array}{l}\text { Erros- } \\
\text { padrão }\end{array}$ & & \\
\hline \multirow[t]{2}{*}{ Valor $(0,60)$} & Confiança & ,780 &, 047 & ,704 & $16,717 * *$ \\
\hline & Satisfação & 211 &, 067 &, 132 & $3,134 * *$ \\
\hline \multirow[t]{3}{*}{ Lealdade $(0,58)$} & Confiança &, 522 &, 068 & ,468 & $7,705^{* *}$ \\
\hline & Valor & ,307 & ,061 & ,305 & $5,021 * *$ \\
\hline & Satisfação & , 104 & 070 &, 064 & 1,473 \\
\hline \multirow[t]{2}{*}{ Lealdade $(0,54)$} & Confiança & ,761 &, 050 & ,683 & $15,204 * *$ \\
\hline & Satisfação &, 168 &, 072 &, 105 & $2,33^{*}$ \\
\hline
\end{tabular}

Fonte: análise de dados.

\section{Discussão e Implicações Gerenciais e Acadêmicas}

A confiança tem relação direta com a lealdade dos clientes?

A mensuração da confiança foi realizada com resultados bastante satisfatórios em todas as três escalas utilizadas (vide resultados nas Tabelas 1 e 2). Essas escalas, depois de consolidadas e analisadas através das regressões múltiplas, permitiram a avaliação do impacto da confiança na lealdade, tanto diretamente, quanto através da mediação do valor percebido. O impacto da confiança na lealdade apresentou-se forte e significante, quando o valor percebido não foi incluído na análise $(\beta=0,68 ; p<0,01)$ e também quando houve a inclusão do valor percebido $(\beta=0,47 ; p<0,01)$, sendo que neste caso houve uma redução razoável na magnitude do impacto $(\Delta=0,24)$, fato semelhante ao identificado por 
Sirdeshmukh et al. (2002). Dessa forma, a hipótese $\mathbf{H}_{1}$ (A lealdade do cliente é positivamente influenciada pela confiança) foi aceita.

Importante observar que a satisfação transacional apresentou-se fraca $(\beta=0,10 ; p<0,01)$, porém significante, quando foi analisada junto à relação confiança-lealdade. O mesmo não ocorreu, quando a variável valor foi incluída na análise; neste caso, a satisfação deixou de ser significante $(\beta=0,06 ; p>0,05)$, o que confirma a proposição de Garbarino e Jonhson (1999) de que para os clientes relacionais (como é o caso da amostra analisada neste trabalho), os grandes responsáveis pela lealdade são a confiança e o comprometimento, e não a satisfação, que seria a responsável pela lealdade de clientes com pouco tempo de relacionamento com a empresa.

Analisadas em conjunto, confiança e satisfação transacional explicaram boa parte do comportamento de lealdade dos clientes $\left(\mathrm{R}^{2}=0,54\right)$, o que demonstra a importância das duas variáveis na explicação desse comportamento de lealdade dos clientes, pois as mesmas representaram mais da metade da variância medida da lealdade.

A confiança tem relação direta com o valor percebido?

O impacto verificado da confiança no valor percebido foi bastante forte e estatisticamente significante ( $\beta=0,70 ; p<0,01)$, demonstrando a importância que a construção dessa confiança tem no relacionamento entre empresa e cliente. Esta importância se concretiza, quando os clientes enxergam que o custo/benefício (isto é, valor percebido) de manter o relacionamento é bastante positivo. Portanto, a hipótese $\mathbf{H}_{2}$ ( $\mathbf{O}$ valor percebido pelo cliente é positivamente influenciado pela confiança) foi aceita.

Há que se destacar também a satisfação transacional que, apesar de estatisticamente significante, apresentou baixo impacto $(\beta=0,13 ; \mathrm{p}<0,01)$. Essas duas variáveis somadas (confiança e satisfação transacional) explicaram cerca de $60 \%$ da variância do valor percebido $\left(\mathrm{R}^{2}=0,60\right)$, mais uma vez um valor bastante próximo ao verificado por Sirdeshmukh et al. (2002) para o contexto de aviação comercial nos EUA $\left(\mathrm{R}^{2}=0,63\right)$, demonstrando a consistência das medidas obtidas e da teoria avaliada através do desenvolvimento e teste do modelo proposto nos dois países.

O valor percebido tem relação direta com a lealdade dos clientes?

Da mesma maneira que o identificado por Sirdeshmukh et al. (2002) para o contexto norte-americano de varejo de vestuário e também para a aviação comercial, o valor percebido pelos clientes no relacionamento com a empresa 
mostrou-se significante para os usuários de Internet Banking brasileiros ( $\beta=0,31$; $\mathrm{p}<0,01)$. A semelhança com o trabalho realizado por aqueles autores no contexto norte-americano deu-se não só na significância dos impactos, mas também na magnitude, que ficou razoavelmente próxima nos dois trabalhos ( $\beta=0,31$ no Brasil e $\beta=0,40$ nos EUA). Dessa forma a hipótese $\mathbf{H}_{4}$ (A lealdade do consumidor em relação ao provedor de serviços é positivamente influenciada pelo valor percebido) foi aceita.

Dois outros destaques merecem ser apresentados. O primeiro diz respeito à variância explicada da variável dependente lealdade, quando medida através das variáveis independentes confiança, valor e satisfação transacional $\left(\mathrm{R}^{2}=0,58\right)$, que, além de ter apresentado uma ótima magnitude, também se mostrou próxima daquela medida no contexto norte-americano para as mesmas variáveis $\left(\mathrm{R}^{2}=0,48\right)$. $\mathrm{O}$ segundo destaque é para o efeito não significante que a variável satisfação transacional apresentou $(\beta=0,06 ; p>0,05)$, o que também aconteceu na pesquisa realizada nos EUA $(\beta=0,09 ; p>0,05)$. Isto demonstra mais uma vez a consistência da teoria que apresenta a confiança, com a mediação de valor percebido, como a variável mais importante para o desenvolvimento da lealdade em clientes relacionais.

O valor percebido é uma variável mediadora na relação entre confiança e lealdade?

Para identificar o papel mediador total da variável "valor" na relação entre confiança e lealdade, três condições devem ser satisfeitas: 1) confiança deve ter impacto direto na lealdade e no valor percebido; 2 ) valor deve ter impacto na lealdade; e 3) o impacto da confiança na lealdade, quando o valor se insere como variável mediadora deve ser praticamente nulo. Analisando os critérios acima, com base nos resultados apresentados na Tabela 5 e na Figura 2, verifica-se que as condições 1 e 2 foram satisfeitas; já a condição 3 não foi satisfeita plenamente, uma vez que o impacto da confiança na variável lealdade, quando o valor foi inserto como variável mediadora baixou significativamente $(\Delta=0,22)$; porém ainda continuou forte e significante ( $\beta=$ $0,47, \mathrm{p}<0,01)$. Dessa forma, valor é variável mediadora parcial e, portanto, hipótese $\mathbf{H 3}$ (Valor percebido é uma variável mediadora da relação confiança-lealdade) foi parcialmente aceita. 
Figura 2: Principais Resultados do Teste do Modelo

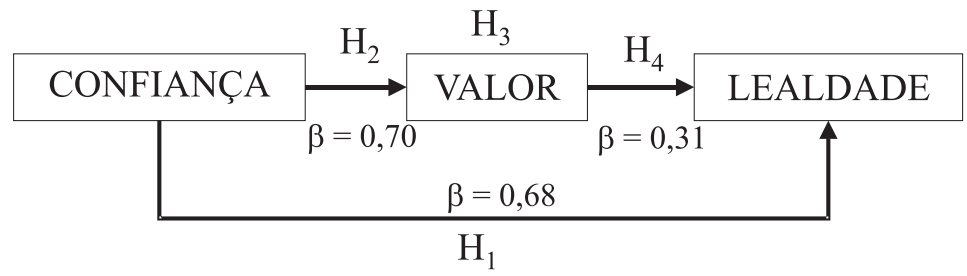

Fonte: análise de dados.

Conclusões, Limitações do Estudo e Sugestões para Pesquisas Futuras

Neste trabalho testou-se um modelo do relacionamento da confiança, valor percebido e lealdade do consumidor final, em trocas relacionais com empresas de serviço. O tamanho da amostra, a abrangência geográfica da pesquisa (todo o Brasil) e os bons resultados obtidos na validação das escalas utilizadas para medir as variáveis em análise permitiram o teste do modelo proposto através da técnica de Modelagem em Equações Estruturais. Este teste do modelo serviu para a avaliação das hipóteses elaboradas no trabalho.

De maneira geral, todas as hipóteses elaboradas sobre as conseqüências da confiança foram aceitas, com exceção da hipótese que apresentava o valor percebido como variável mediadora do relacionamento entre a confiança e lealdade dos clientes, que foi parcialmente aceita. Os resultados obtidos podem ser analisados sob duas óticas principais: a contribuição para a teoria de marketing e as conseqüências gerenciais.

Em termos teóricos, os resultados sustentam alguns achados recentes a respeito da relação entre confiança, valor, satisfação transacional e lealdade. Em primeiro lugar, a importância do desenvolvimento da confiança do cliente na consolidação das trocas relacionais foi reforçada, conforme já fora preconizado por Morgan e Hunt (1994) e Garbarino e Johnson (1999).

Em segundo lugar, para os clientes relacionais, os resultados indicam que a satisfação transacional tem importância secundária em relação à confiança e ao valor percebido na formação da lealdade dos clientes, o que também já fora identificado em outro contexto por Sirdeshmukh et al. (2002). Uma terceira e importante constatação teórica diz respeito à importância crescente que os estudos sobre valor percebido pelo cliente tem recebido em marketing. Os achados 
empíricos reforçam a teoria de que, quando se analisam trocas relacionais, não se pode restringir a análise apenas à confiança ou ao valor percebido como antecedentes da lealdade. Pelo contrário, os resultados reforçam o papel concomitante desses dois construtos na formação da lealdade do cliente.

Uma quarta e última constatação teórica pode ser feita a respeito da importância da validação de modelos em diferentes contextos de pesquisa. O teste bem sucedido de um modelo com características semelhantes por Sirdeshmukh et al. (2002), em outro país, com cultura diferente da brasileira e em outros setores econômicos, e os vários resultados semelhantes obtidos em termos de magnitude e significância estatística são forte indicador da validade da teoria analisada.

A partir do desenvolvimento e teste do modelo, pode-se, ainda, fazer algumas proposições a respeito da atuação dos gerentes de marketing da área bancária, em sua gestão do relacionamento com seus consumidores. Verificou-se que os clientes que já desenvolveram longo relacionamento com a empresa atribuem maior importância à confiança que depositam nessa empresa; e que essa confiança, atribuída à empresa, posteriormente se transforma em lealdade. Essas constatações não sugerem que os gerentes devam atribuir pouca importância à satisfação de seus consumidores, mas sim que, para esse grupo de clientes já cativos, a satisfação não é a percepção mais preponderante para suas decisões de permanecerem mantendo negócios com a empresa.

Outra importante constatação que pode ajudar na definição de estratégias de relacionamento dos bancos é que a percepção de custo/benefício (em outras palavras, valor percebido) é uma preocupação assaz relevante para a manutenção desses relacionamentos. Assim, a estratégia de preços (incluindo-se aí tarifas sobre serviços, taxas de juros para empréstimos ou para investimentos) devem ser definidas sob essa ótica de custo/benefício para o cliente. Um possível exemplo de aplicação gerencial dos resultados do modelo é este: aqueles clientes que pagam mais tarifas, por utilizarem mais serviços, consideram importante que o banco melhore a contrapartida desses pagamentos, como a remuneração de seus investimentos.

Por fim, deve-se ressaltar que, como toda a pesquisa, esta apresenta algumas importantes limitações que podem ser minimizadas em trabalhos futuros. Algumas limitações desse trabalho foram a coleta de dados em corte transversal (isto é, em apenas um dado momento), quando um estudo longitudinal é mais apropriado para a determinação de relações de causa e efeito entre comportamentos de clientes. Outras limitações que podem ser citadas são: a seleção da amostra de clientes entrevistados em apenas uma empresa de serviço (mesmo tendo abrangência nacional e sendo uma das principais organizações do mercado em 
que atua) e a característica em comum dos clientes de serem todos usuários de Internet Banking, todas elas possíveis geradoras de viés nos resultados.

Trabalhos de pesquisa que buscassem amostras longitudinais, com dados coletados utilizando-se mais de um método e a comparação de resultados entre clientes de várias empresas e setores econômicos seriam bastante adequados para proporcionar maior validade externa às relações entre confiança, valor e lealdade aqui analisadas; relações estas da maior importância para toda empresa que hoje procura estabelecer um relacionamento com seus clientes.

\section{Artigo recebido em 20.08.2003. Aprovado em 23.11.2004.}

\section{Notas}

${ }^{1}$ As escalas adaptadas do trabalho de Sirdeshmukh et al. (2002) e utilizadas neste artigo foram obtidas antes da publicação daquele trabalho no Journal of Marketing, a partir de working paper redigido em 2000 e enviado para os autores deste artigo pelos autores daquele trabalho, aos quais desde já agradecemos.

\section{ReferÊnCIAS Bibliográficas}

BAGOZZI, R.

Marketing as exchange. Journal of Marketing, New York, Iss. 4, v. 39, p. 32-39, oct. 1975.

CHANG, T.;

WILDT, A. P.

Product Information, and Purchase Intention: an empirical study. Journal of the Academy of Marketing Science, Greenvale, Iss. 1,v. 22, p. 1627, winter 1994.

\section{CROSBY,L.;}

STEPHENS, N.

Effects of relationship on satisfaction, retention, and prices on the life insurance industry. Journal of Marketing Research, Chicago, Iss. 4, v. 24, p. 404-411, nov. 1987.
DICK, A.;

BASU, K.

Customer Loyalty: Toward an Integrated Conceptual Framework. Journal of the Academy of Marketing Science, Greenvale, Iss. 2, v. 22, n. 2, p. 99-113, spring 1994.

DODDS, W.;

MONROE, K.;

GREWAL, D.

Effects of price, brand, and store information on buyers' product evaluations. Journal of Marketing Research, Chicago, Iss. 3, v. 28, p. 307-319, aug. 1991. 
DONEY, P.;

CANNON, J.

An examination of the nature of trust in Buyer-Seller relationships. Journal of Marketing, Chicago, Iss. 2, v. 61, p. 35-51, apr. 1997.

DWYER, R.;

SCHURR, P.;

$\mathrm{OH}, \mathrm{S}$.

Developing Buyer-Seller relationships. Journal of Marketing, Chicago, Iss. 2, v. 51, p. 11-27, apr. 1987.

\section{GANESAN, S.;}

HESS, R.

Dimensions and levels of trust: implications for commitment to a relationship. Marketing Letters, USA, v.8, n. 4, p. 439-448, oct. 1997.

GASSENHEIMER, J.;

HOUSTON, F.;

DAVIS, C.

The role of economic value, social value, and perceptions of fairness in interorganizational relationship retention decisions. Journal of the Academy of Marketing Science, Greenvale, Iss. 4, v. 26, n.4, p. 322337, fall 1998.

GARBARINO, E.;

JOHNSON, M.

The different roles of satisfaction, trust and commitment for relational and transactional consumers. Journal of Marketing, Chicago, Iss. 2, v. 63, p. 70-87, apr. 1999.

\section{GEYSKENS, I.;}

STEENKAMP, J.;

KUMAR, N.

Generalizations about trust in marketing channels relationships using meta-analysis. International
Journal of Research in Marketing, Amsterdam, Iss. 3, v. 15, p. 223-248, july 1998.

GRISAFFE, D.;

KUMAR, A.

Antecedents and consequences of customer value: testing an expanded framework. Marketing Science Institute Working Paper, Massachusetts, \#98-107, may 1998.

\section{GROONRÖOS, C.}

Services Management and Marketing - a customer relationship management approach.

2. ed. England: Wiley, 2000. p. 394.

HAIR, J. et al.

Multivariate data analysis. 5. ed. Upper Saddle River: Prentice-Hall, 1998.730p.

KRAMER, R.

Trust and distrust in organizations: emerging perspectives, enduring questions. Annual Review of Psychology, Palo Alto, v. 50, p. 569598, feb. 1999.

MOORMAN, C.;

DESHPANDE, R.;

ZALTMAN, G.

Factors affecting trust in market research relationships. Journal of Marketing, Chicago, Iss. 1, v.57, p. 81-101, jan. 1993.

\section{MORGAN, R.;}

HUNT, S.

The commitment-trust theory of relationship marketing, Journal of Marketing, Chicago, Iss. 3, v. 58, p. 20-38, july 1994. 
MURRAY, K.

A test of services marketing theory: consumer information acquisition activities, Journal of Marketing, Chicago, Iss. 1, v. 55, p. 10-25, jan. 1991.

\section{NEAL, W.}

Satisfaction is Nice, but value drives loyalty. Marketing Research, Chicago, Iss. 1, p. 21-23, spring, 1999.

\section{OLIVER, R.}

Satisfaction: a behavioral perspective on the consumer. New York: Irwin / McGraw-Hill, 1997. 1. ed. 432p.

Whence customer loyalty? Journal of Marketing, Chicago, Special Issue, v. 63, special issue, p. 33-44, 1999.

\section{PARASURAMAN, A.;}

GREWAL, D.

The impact of technology on the quality-value-loyalty chain: a research agenda. Journal of the Academy of Marketing Science, Greenvale, Iss. 1, vol. 28, n. 1, p. 168174, winter 2000.

\section{ROTTER, J.}

A new scale for the measurement of interpersonal trust. Journal of Personality, United Kingdom, Iss. 4, v. 35, p. 651-665, dec. 1967.

\section{ROUSSEAU, D. et. al.}

Not so different After All: a cross discipline view of trust. Academy of Management Review, Briarcliff Manor, Iss. 3, v. 23, n. 3, p. 393-404, jul. 1998.

\section{SINGH, J.}

Understanding the Structure of Consumers Satisfaction Evaluations of Service Delivery, Journal of the
Academy of Marketing Science, Greenvale, Iss. 3, v. 19, n. 3, p. 223244, summer 1991.

SIRDESHMUKH, D.

Agency and Trust Mechanisms in Relational Exchanges. Journal of the Academy of Marketing Science, Greenvale, Iss. 1, v. 28, p. 150-167, winter 2000.

\section{SIRDESHMUKH, D;}

SINGH, J.;

SABOL, B.

Consumer Trust, Value, and Loyalty in Relational Exchanges. Journal of Marketing, Chicago, Iss. 1, v. 66, p. 15-37, jan. 2002.

SZYMANSKI, D.;

HENARD, D.

Customer Satisfaction: a metaanalysis of the empirical evidence. Journal of the Academy of Marketing Science, Greenvale, Iss. 1, v. 29, p. 16-35, winter 2001.

\section{URBAN, G.;}

SULTAN, F.;

QUALLS, W.

Placing Trust at the Center of Your Internet Strategy. Sloan Management Review, Cambridge, Iss. 1, v. 42, p. 3948, fall 2000.

\section{VANDERMERWE, S.}

How Increasing Value to Customers Improves Business Results. Sloan Management Review, Cambridge, Iss. 1, v. 42, p. 27-37, fall 2000. 
ZEITHAML, V.

Consumer Perceptions of Price, Quality, and Value: A means-end Model and Synthesis of Evidence, Journal of Marketing, Chicago, Iss. 3, v. 52, p.2-22, July 1988.
ZEITHAML, V.;

BERRY,L.;

PARASURAMAN, A.

The Behavioral Consequences of Service Quality. Journal of Marketing, Chicago, Iss. 2, v. 60, p. 2-22, apr. 1996. 